\title{
Rogue-waves generation in the terahertz region
}

\author{
R.M. Rozental, N.S.Ginzburg, A.M. Malkin, A.S. Sergeev, V.Yu. Zaslavsky, I.V. Zotova \\ Institute of Applied Physics RAS, Nizhny Novgorod, Russia, rrz@appl.sci-nnov.ru
}

One of the promising schemes of high-power terahertz radiation generators are gyrotrons with planar configuration of interaction space [1]. In such a configuration, by reducing the distance between the plates of the cavity, it is possible to obtain a large current parameter sufficient for occurrence of rogue waves - ultrashort pulses with a peak power exceeding the power of the unperturbed electron beam [2].

For description of rogue waves in gyrotron the self-consistent time-domain model was used:

$i \frac{\partial^{2} a}{\partial Z^{2}}+\frac{\partial a}{\partial \tau}=\frac{i I_{0}}{2 \pi} \int_{0}^{2 \pi} \frac{\hat{p}_{\perp}}{\hat{p}_{\|}} d \theta_{0}$

$\left[\frac{\partial}{\partial Z}+\frac{g^{2}}{4} \frac{\partial}{\partial \tau}\right] p_{\perp}+i \frac{p_{\perp}}{p_{\|}}\left(\Delta-1+\left|p_{\perp}\right|^{2}+\frac{p_{\|}^{2}-1}{g^{2}}\right)=i \frac{a}{\hat{p}_{\|}}+\frac{\beta_{\perp 0}^{2}}{2} \frac{\partial a}{\partial Z}$

$\left[\frac{\partial}{\partial Z}+\frac{g^{2}}{4} \frac{\partial}{\partial \tau}\right] p_{\|}=-g^{2} \frac{\beta_{\perp 0}^{2}}{2} \operatorname{Re}\left(\frac{\partial a}{\partial Z} \frac{p_{\perp}{ }^{*}}{p_{\|}}\right)$

where $a(Z, \tau)$ - normalized amplitude of the operating wave; $Z, \tau-$ normalized coordinate and time; $p_{\perp, \|}-$ normalized particle moments, $I_{0}$ - current parameter, $\Delta$ - initial mismatch between the wave cutoff frequency, $g$ - initial pitch-ratio.

In simulations we assumed that the $300 \mathrm{GHz}$ gyrotron excited by the planar electron beam with energy of about $200 \mathrm{keV}$, current density of about $2 \mathrm{kA} / \mathrm{cm}$ and pitch-factor of about 1.0 at the lowest TE mode of the planar waveguide. With this parameters the value of the dimensionless current parameter $I_{0} \approx 1$, which is which is enough for the radiation of rogue waves.

All processes were analyzed over a time interval of several tens of nanoseconds, which corresponds to the typical pulse duration in electron guns based on explosive emission cathodes. Simulation showed that in a sufficiently wide range of magnetic fields in the system, it is possible to generate rogue waves with a peak power of several hundred megawatts (Fig.1).

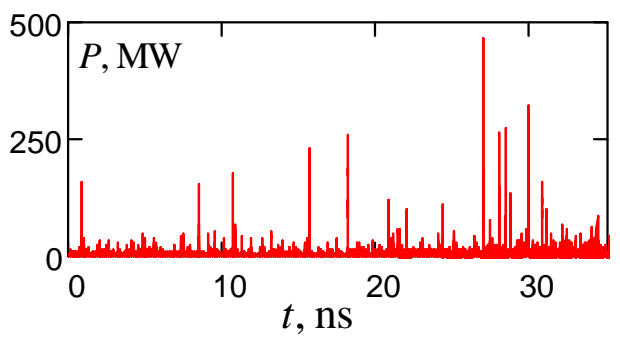

Fig. 1. The time dependence of the output power for simulation of planar gyrotron based on the averaged equations.
The obtained results have been confirmed by direct PIC simulations at KARAT code [3]. In the simulation, pulses of a duration of the order of 10 ps were obtained with a peak power reaching hundreds of megawatts (Fig.2a). The total width of the emission spectrum reached $150 \mathrm{GHz}$ (Fig. 2b).
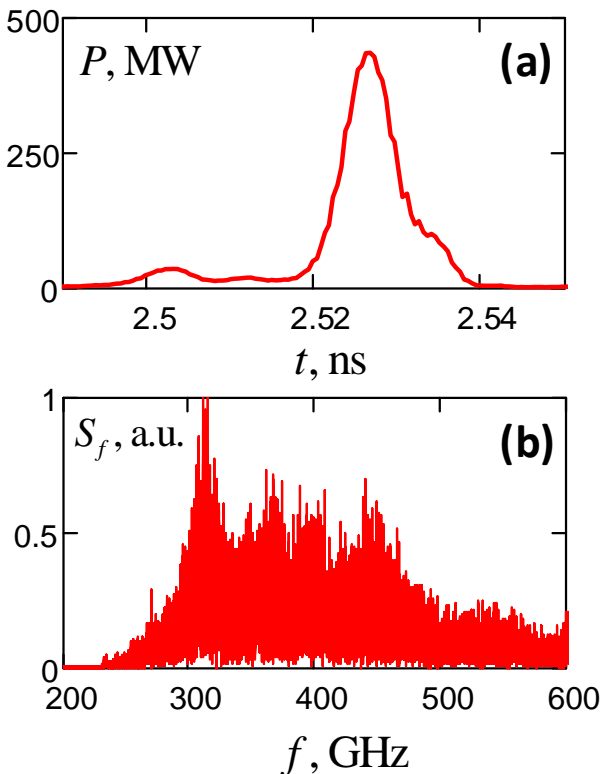

Fig. 2. Results of PIC simulation: (a) - a typical form of an rogue wave; (b) is the total spectrum of the output radiation.

This research was performed within the framework of the RFBR project No. 17-08-01077.

\section{References}

1. Zaslavsky, V.Yu., Ginzburg, N.S., Glyavin, M.Yu., Zheleznov, I.V., Zotova, I.V. Three-dimensional particle-incell modeling of terahertz gyrotrons with cylindrical and planar configurations of the interaction space // Phys. Plasmas. 2013. V. 20. Art.no. 043103

2. Ginzburg, N.S., Rozental, R.M., Sergeev, A.S., Fedotov, A.E., Zotova, I.V., Tarakanov, V.P. Generation of Rogue Waves in Gyrotrons Operating in the Regime of Developed Turbulence // Phys. Rev. Lett. 2017. V.119. Art. no. 034801.

3. Tarakanov, V.P. Code KARAT in simulations of power microwave sources including Cherenkov plasma devices, vircators, orotron, E-field sensor, calorimeter etc. // EPJ Web of Conferences. 2017. V. 331. Art.no. 04024. 